\title{
NOTES
}

\section{Degradation of Ultrahigh Molecular Weight Poly(styrene-co-m-divinylbenzene)s as Network-Polymer Precursors in SEC Columns}

\author{
Akira Matsumoto, ${ }^{\dagger}$ Hiroaki Oe, and Hiroyuki Aota \\ Department of Applied Chemistry, Faculty of Engineering and High Technology Research Center, \\ Kansai University, Suita, Osaka 564-8680, Japan
}

(Received November 2, 2001; Accepted February 4, 2002)

\begin{abstract}
KEY WORDS Degradation / SEC Column / Styrene / $m$-Divinylbenzene / Ultrahigh Molecular Weight / Network-Polymer Precursor /
\end{abstract}

As an extension of our continuing studies concerned with the mechanistic discussion of the three-dimensional network formation in the freeradical polymerization and copolymerization of multivinyl compounds, ${ }^{1}$ the preceding article ${ }^{2}$ dealt with the crosslinking copolymerization of styrene $(\mathrm{St})$ with $m$-divinylbenzene ( $m$-DVB) as a most typical monovinyl/divinyl system. Thus, the reasons for the greatly delayed gelation from Flory-Stockmayer gelation theory (FS theory) ${ }^{3}$ have been discussed extensively in order to clarify the crosslinking polymerization mechanism of multivinyl compounds. ${ }^{1}$ The actual gel point should be close to the theoretical one if the experiment were done under the polymerization conditions in which the polymer concentration, at the theoretical gel point, would be high enough to reduce the significance of a thermodynamic excluded volume effect as a primary factor and, furthermore, the intramolecular crosslinking preceded by the intermolecular crosslinking could be suppressed. Our interpretations are verified for monomethacrylate/dimethacrylate, ${ }^{4,5}$ monoacrylate/diacrylate, ${ }^{6}$ and $\mathrm{St} / m$-DVB ${ }^{2}$ copolymerizations, in which the results obtained are discussed in terms of ideal network formation according to FS theory.

In a series of these investigations, the detailed pursuit of the variation of molecular-weight distribution (MWD) curves with conversion is inevitable for a full understanding of crosslinking polymerization because the MWD curves should rapidly broaden towards a higher molecular weight side with conversion as a result of exclusive occurrence of intermolecular crosslinking reaction leading to ideal network formation. ${ }^{3,7,8}$ Here it is worthy to note that a size-exclusion chromatography (SEC) is commonly used to determine

${ }^{\dagger}$ To whom correspondence should be addressed. the molecular weight and the MWD of resulting prepolymers. However, as a technique it is not always applicable to our crosslinking polymerization systems because the structure of prepolymer changes from a linear to a branched form with progressing polymerization and, moreover, the content of ring or loop structure introduced into the linear portion or primary chain and the content of multiple crosslink or network structure in the branched form depend on the polymerization conditions. On the other hand, SEC equipped with a multiangle laser light scattering (MALLS) device (SECMALLS) is expected to be a very useful and efficient tool in characterizing our prepolymers because the use of MALLS, as an absolute detector, enables the molecular weight and MWD to be determined directly without any calibration. ${ }^{5}$

During the progress of our investigation, we noticed that the degradation of poly(St-co-m-DVB) prepolymer of ultrahigh molecular weight obtained at the conversion close to the gel point occurred significantly in SEC columns. That is, the molecular weights determined by both SEC-MALLS and light scattering (LS) measurements were compared in detail. Although both results were in a good agreement within experimental errors for poly(St-co-m-DVB) prepolymers of moderate molecular weight less than one million, the discrepancy between both measurements became greater with further increment of molecular weight, i.e., with the progress of crosslinking.

Here it should be recalled that the chain scission in SEC columns has been observed for poly(St) of ultrahigh molecular weight. ${ }^{9-11}$ Barth et al. ${ }^{11}$ have reviewed the polymer shear degradation in SEC and guidelines involving operational parameters are presented. Thus, high molecular weight polymers are fairly sensitive to shear forces which can lead to chain scission. Be- 
cause of velocity gradients generated during flow in SEC columns, a polymer becomes extended. Bonds near the middle of the chain become stretched and can rupture if shear rates are great enough. As the end segments of a chain maintain their coiled shape, the maximum strain is usually focused near the center of the polymer. In general, the most probable sites of stress concentration on the polymer chain for the application of shear force are: (1) side chain linkages to main chain, i.e., branch points, (2) crosslink points in networks, (3) points of inclusion of heteroatoms and quaternary carbon atoms, and (4) dissymmetry between adjacent atoms which promotes rupture of the chain by stretching forces. The former two points may be closely related to the degradation of our network-polymer precursors.

By considering the above discussion, we tried to clarify the correlation of the specific degradability with the characteristic features of vinyl-type network-polymer precursors including primary chain length, branched structure, and multiple crosslink or network structure. This kind of the degradation of network-polymer precursors in SEC columns may be significantly related to the discussion not only of network formation, but also of branching by using SEC. ${ }^{12-14}$

\section{EXPERIMENTAL}

St as monomer, 2,2'-azobisisobutyronitrile (AIBN) as initiator, and toluene as solvent were purified by conventional methods. $m$-DVB, supplied by Nissei Chemical Industry Co., Ltd., consisting of $94.0 \%$ of $m$-DVB, $5.2 \%$ of $p$-DVB, and $0.8 \%$ of ethylstyrene, was used without further purification.

Polymerization was conducted as described previously. $^{2}$

SEC-MALLS measurements were carried out at $40^{\circ} \mathrm{C}$ in tetrahydrofuran (THF) using a Shodex GPC KF- $806 \mathrm{~L} \times 5$ columns, at polymer concentrations $0.05-$ $0.1 \%(\mathrm{w} / \mathrm{v})$ and flow rate $1 \mathrm{~mL} \mathrm{~min}^{-1}$. The MALLS device was a DAWN model F (Wyatt Technology Corp.) where the laser beam, of wavelength $632.8 \mathrm{~nm}$, was focused on a $67 \mu \mathrm{L}$ flow cell.

LS measurements were carried out in THF at $30^{\circ} \mathrm{C}$ with an Otsuka Electronics DLS-700 dynamic light scattering spectrophotometer over the angular range between 30 and $150^{\circ}$, using unpolarized light of wavelength $632.8 \mathrm{~nm}$. The optical clarification of the polymer solution was done carefully by filtration using $1 \mu$ micropore filter.

\section{RESULTS AND DISCUSSION}

In the preceding article, ${ }^{2}$ we have discussed the applicability of FS theory ${ }^{3}$ for the crosslinking copolymerizations of St with $m$-DVB. The higher the theoretical gel point, the smaller the ratio of the actual gel point to the theoretical one. In the presence of $0.17 \mathrm{~mol} \%$ of $m$-DVB, its ratio reached 1.3 , close to unity, supporting the good applicability of FS theory. In addition, the more tailed MWD curves are observed up to $39.2 \%$ conversion as a result of predominant occurrence of intermolecular crosslinking reaction ${ }^{3,7,8}$ approaching to ideal network formation governed by FS theory.

As an extension of the above work, we tried to characterize further the resulting prepolymers by LS measurements. Then, we noticed that the degradation of poly(St-co-m-DVB) prepolymer of ultrahigh molecular weight occurred significantly in SEC columns since the molecular weights of prepolymers determined by LS did not coincide with those determined by SECMALLS. Here it worthy to note that both measurements by LS and SEC-MALLS give absolute values of weight-average molecular weight, $\bar{M}_{\mathrm{w}}$ (LS) and $\bar{M}_{\mathrm{w}}$ (SEC-MALLS), respectively. The LS measurements were done directly for the solutions of prepolymers in THF without passing through the SEC columns and thus, no chance of the degradation of poly(St-co-mDVB) of ultrahigh molecular weight in SEC columns would be ensured as is completely different from the case of the SEC-MALLS measurements. This finding prompted us to examine in more detail the degradation of poly(St-co-m-DVB) prepolymers as vinyl-type network-polymer precursors.

Earlier, Slagowski et al. ${ }^{9}$ have reported that the molecular weight of the original polymer $\left(M_{\mathrm{W}}=4.4 \times\right.$ $10^{7}$ ) had been decreased to $1.9 \times 10^{7}$. In this connection, we prepared a linear poly(St) of ultrahigh molecular weight $\left(\bar{M}_{\mathrm{w}}(\mathrm{LS})=8.1 \times 10^{6}\right)$ by the emulsion polymerization and then, the poly(St) was subjected to SECMALLS measurement to give $\bar{M}_{\mathrm{w}}$ (SEC-MALLS) $/ \bar{M}_{\mathrm{w}}$ (LS) value of 0.37 .

Then, we tried to check the degradation of the prepolymers of ultrahigh molecular weight of more than one million obtained in the copolymerization of $\mathrm{St}$ with 0.25 and $1 \mathrm{~mol} \%$ of $m$-DVB using $0.04 \mathrm{~mol} \mathrm{~L}^{-1}$ of AIBN at $60^{\circ} \mathrm{C}$; the gel points were estimated to be 48.8 and $21.5 \%$, respectively. ${ }^{2}$ Figure 1 shows the conversion dependence of weight-average molecular weight, $\bar{M}_{\mathrm{w}}$ (LS), of the prepolymer determined by LS; the prepolymer of a higher $\bar{M}_{\mathrm{W}}$ value was obtained for the copolymerization with a higher $m$-DVB mole percent 


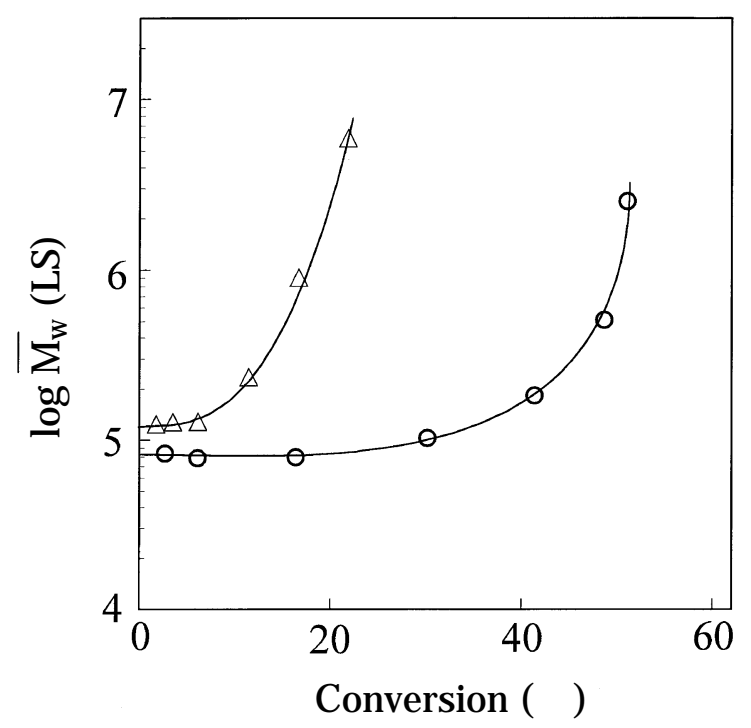

Figure 1. Dependence of $\bar{M}_{\mathrm{w}}$ (LS) of the prepolymer determined by LS on conversion for the bulk copolymerizations of St with $(\bigcirc) 0.25$ and $(\triangle) 1 \mathrm{~mol} \%$ of $m$-DVB using $0.04 \mathrm{~mol} \mathrm{~L}^{-1}$ of AIBN at $60^{\circ} \mathrm{C}$.

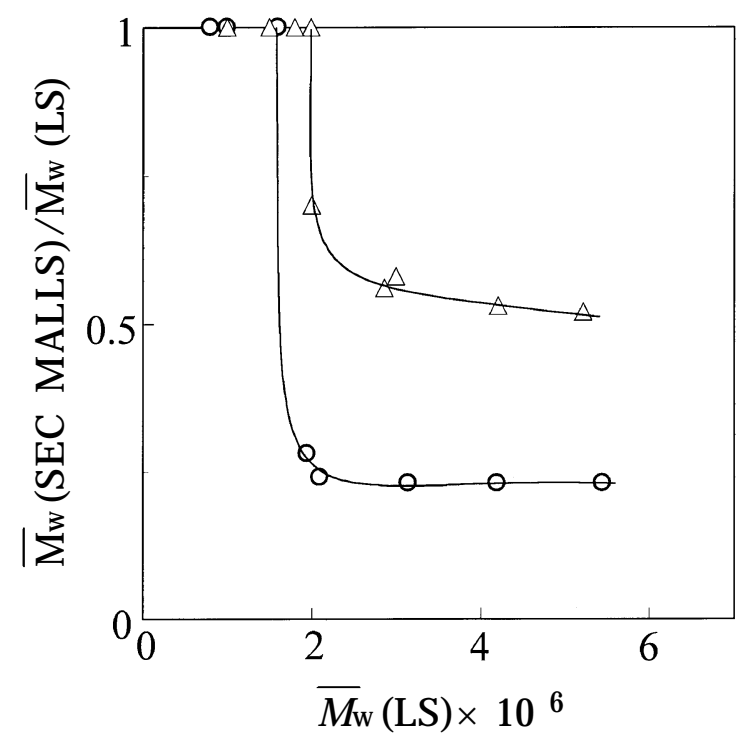

Figure 2. Degradation of poly(St-co-m-DVB) prepolymers (see Figure 1).

in a feed monomer mixture. Figure 2 shows the plots of weight-average molecular weight ratio $\bar{M}_{\mathrm{w}}$ (SECMALLS) $/ \bar{M}_{\mathrm{w}}$ (LS) vs. $\bar{M}_{\mathrm{w}}$ (LS); the ratio became smaller with decreasing the mole percentage of $m$-DVB in the feed monomer. Since $\bar{M}_{\mathrm{w}}$ (SEC-MALLS) $/ \bar{M}_{\mathrm{w}}$ (LS) will be a measure of degradation of prepolymer in SEC columns, the degradation became marked with a decrease in the crosslinker content, i.e., from 1 to 0.25 mol $\%$ of $m$-DVB. Here it should be recalled that the ratio of the actual gel point to the theoretical one was estimated to be 1.8 and $5.0,{ }^{2}$ respectively, in the copolymerizations of St with 0.25 and $1 \mathrm{~mol} \%$ of $m$-DVB as a reflection of delayed gelation from FS theory. ${ }^{3}$ The oc-

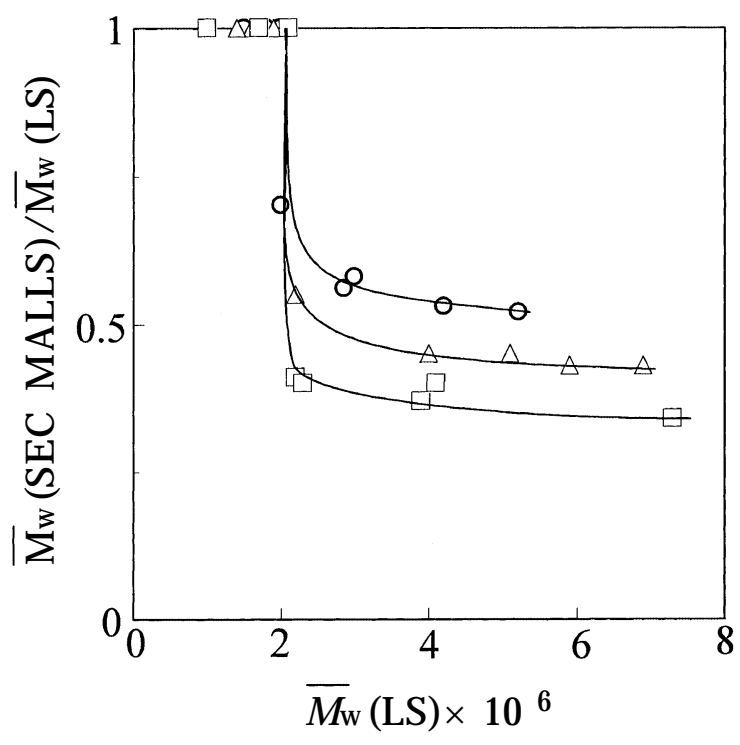

Figure 3. Degradation of poly(St-co-m-DVB) prepolymers obtained in the copolymerization of St with $1 \mathrm{~mol} \%$ of $m$-DVB in the absence $(\bigcirc)$ and presence of $(\triangle) 2 \times 10^{-3}$ and (口) $3.5 \times 10^{-3} \mathrm{~mol}$ $\mathrm{L}^{-1}$ of lauryl mercaptan using $0.04 \mathrm{~mol} \mathrm{~L}^{-1}$ of AIBN at $60^{\circ} \mathrm{C}$. Here the primary chain lengths, $\bar{P}_{\mathrm{w}, 0}$, were estimated to be 1190,650 , and 305 , respectively, by the extrapolation of the conversion dependence of weight-average molecular weight of the prepolymer to zero conversion.

currence of intramolecular crosslinking leading to multiple crosslink became remarkable with increasing the mole percentage of $m$-DVB in the feed. ${ }^{2}$ Conclusively, the degradation of resulting poly(St-co-m-DVB) prepolymers became marked with reduced occurrence of intramolecular crosslinking or the decrement of multiple crosslink or network structure, i.e., with approaching to ideal network formation governed by FS theory. ${ }^{2}$

Next, we tried to examine the dependence of degradation on primary chain length of resulting prepolymers because the shorter primary chain inevitably leads to more highly branched structure in order to produce the prepolymer of ultrahigh molecular weight. Thus, three kinds of poly(St- $c o-m$-DVB) prepolymers of different primary chain lengths were prepared as follows: $\mathrm{St} / m$-DVB (molar ratio 99:1) copolymerizations were conducted in bulk in the absence and the presence of $2 \times 10^{-3}$ and $3.5 \times 10^{-3} \mathrm{~mol} \mathrm{~L}^{-1}$ of lauryl mercaptan as chain transfer agent using $0.04 \mathrm{~mol} \mathrm{~L}^{-1}$ of AIBN at $60^{\circ} \mathrm{C}$; the primary chain lengths, $\bar{P}_{\mathrm{w}, 0}$, of resulting prepolymers were estimated to be 1190,650 , and 305 , respectively. Figure 3 shows the plots of $\bar{M}_{\mathrm{w}}$ (SECMALLS) $/ \bar{M}_{\mathrm{w}}$ (LS) vs. $\bar{M}_{\mathrm{w}}$ (LS). Obviously, the degradation of prepolymers became more remarkable with decreased primary chain length, i.e., with the increment of branching.

Finally, two types of prepolymers containing different amounts of loop and network structures were pre- 
Table I. Comparison of actual gel points with theoretical ones for the copolymerizations of St with $m$-DVB

\begin{tabular}{|c|c|c|c|c|c|}
\hline$m$-DVB & \multirow{2}{*}{ Dilution } & \multirow{2}{*}{$\bar{P}_{\mathrm{w}, 0} \times 10^{-3}$} & \multicolumn{2}{|c|}{ Gel point $(\%)$} & Actual G.P. ${ }^{c}$ \\
\hline $\mathrm{mol} \%$ & & & Actual $^{\mathrm{b}}$ & Theoretical $^{\mathrm{d}}$ & Theoretical G.P. $^{\mathrm{d}}$ \\
\hline 3 & $1 / 3$ & 0.41 & $39.2(39.1)^{\mathrm{c}}$ & 4.1 & 9.5 \\
\hline 5 & $1 / 5$ & 0.28 & $51.5(51.2)^{\mathrm{c}}$ & 3.7 & 13.8 \\
\hline
\end{tabular}

${ }^{\mathrm{a}}$ In toluene, $[\mathrm{AIBN}]=0.04 \mathrm{~mol} \mathrm{~L}-1,60^{\circ} \mathrm{C} .{ }^{\mathrm{b}}$ Obtained on monomer basis. ${ }^{\mathrm{c}}$ Obtained as the viny1 group conversion calculated by assuming equal reactivity of St and $m$-DVB viny1 groups. ${ }^{\mathrm{d}}$ Theoretical gel point: $\alpha_{\mathrm{c}}=(1 / \rho)\left(\bar{P}_{\mathrm{w}, 0}-1\right)^{-1}$.

pared by the solution copolymerizations of St with 3 and $5 \mathrm{~mol} \%$ of $m$-DVB, as rather high amounts of crosslinker, in toluene at dilutions of $1 / 3$ and $1 / 5$, respectively. Table I summarizes the comparison of actual gel points with theoretical ones; the gelation occurred quite later than predicted as a reflection of an extensive occurrence of intramolecular cyclization and crosslinking leading to the enhanced formation of loop and network structures. The degradation of the prepolymers obtained at a dilution of $1 / 5$ became less remarkable compared with those at a dilution of $1 / 3$, suggesting the reduced occurrence of degradation by the incorporation of loop and network structures into the prepolymer. Here, it is worthy to note that the primary chain length $\bar{P}_{\mathrm{w}, 0}$ was lower for the prepolymers obtained at a dilution of $1 / 5$, in spite of the above result that the shorter primary chain led to the promoted occurrence of degradation.

In conclusion, the degradation of ultrahigh molecular weight poly(St-co-m-DVB)s obtained at the conversion close to the gel point occurred significantly in SEC columns. The specific degradability was correlated to the characteristic features of vinyl-type networkpolymer precursors including primary chain length, branched structure, and multiple crosslink or network structure.

\section{REFERENCES}

1. A. Matsumoto, Adv. Polym. Sci., 123, 41 (1995).

2. A. Matsumoto, Y. Kitaguchi, and O. Sonoda, Macromolecules, 32, 8336 (1999).

3. W. H. Stockmayer, J. Chem. Phys., 12, 125 (1944).

4. A. Matsumoto, S. Okuno, and H. Aota, Macromol. Symp., 93, 1 (1995).

5. A. Matsumoto, A. Okamoto, S. Okuno, and H. Aota, Angew. Makromol. Chem., 240, 275 (1996).

6. A. Matsumoto and A. Taniguchi, Polym. J., 31, 711 (1999).

7. M. Oiwa, Nippon Kagaku Zasshi, 76, 684 (1955).

8. H. Tobita, Macromol. Theory Simul., 7, 225 (1998).

9. E. L. Slagowski, L. J. Fetters, and D. McIntyre, Macromolecules, 7, 394 (1974).

10. D. McIntyre, A. L. Shih, J. Savoca, R. Seeger, and A. MacArthur, ACS Symp. Ser., 245, 227 (1984).

11. H. G. Barth and F. J. Carlin, J. Liq. Chromatogr., 7, 1717 (1984).

12. M. Kurata, H. Okamoto, and M. Iwama, Polym. J., 3, 739 (1972).

13. W. S. Park and W. W. Graessley, J. Polym. Sci., Polym. Phys. Ed., 15, 85 (1977).

14. D. E. Axelson and W. C. Knapp, J. Appl. Polym. Sci., 25, 119 (1980). 